\title{
EI SEO en la empresa periodística: percepciones y elementos clave para su adopción en las redacciones
}

\section{SEO in the journalistic company: perceptions and key elements for its adoption in writing}

Carlos Lopezosa. Universitat Pompeu Fabra. España.

carlos.lopezosa@upf.edu

$[\underline{\mathrm{CV}}]$ (1) $\mathrm{G} \mathrm{B}_{\mathrm{C}} \mathrm{F}$

Magdalena Trillo-Domínguez. Universidad de Granada. España.

mtrillo@ugr.es

$[\mathrm{CV}](\mathrm{C}$

Lluís Codina. Unıversidad Pompeu Fabra. España.

lluis.codina@upf.edu

$[\underline{\mathrm{CV}}]$ (1) $\mathrm{G} \mathrm{R}^{\mathrm{V}} \mathrm{F}$

Marga Cabrera Méndez. Universitat Politècnica de València. España.

mcabrera@upv.es

$[\underline{\mathrm{CV}}]$ (1) G

Este artículo es producto del proyecto de Investigación titulado "Narración interactiva y visibilidad digital en el documental interactivo y el periodismo estructurado", referencia RTI2018-095714-B-C21 (MICINN/FEDER), financiado por la Dirección General de Investigación y Gestión del Plan Nacional de $I+D+i$, del Ministerio de Ciencia e Innovación.

\section{Cómo citar este artículo / Referencia normalizada}

Lopezosa, C., Trillo-Domínguez, M., Codina, L. y Cabrera Méndez, M. (2021). El SEO en la empresa periodística: percepciones y elementos clave para su adopción en las redacciones. Revista Latina de Comunicación Social, 79, 27-45. https://www.doi.org/10.4185/RLCS-2020$\underline{1487}$

\section{RESUMEN}

Introducción. Se analiza la importancia que dan los profesionales de los medios de comunicación al posicionamiento en buscadores en una redacción periodística y sus diferentes dimensiones. En concreto, se estudia la percepción de los profesionales del sector periodístico tras la reciente aplicación de estrategias de Search Engine Optimization (SEO) en el Diario de Sevilla, cibermedio del Grupo Joly, con tradición en papel y que, a partir de mayo de 2018, creó un departamento específico de posicionamiento web que sería su proyecto piloto para la paulatina transformación del resto de sus cabeceras. Metodología. Para el desarrollo de esta investigación se ha aplicado la observación participante, las entrevistas semiestructuradas y el análisis de frecuencia de términos del manual interno de la empresa sobre estrategias de posicionamiento en buscadores. Conclusiones $\mathbf{y}$ discusión. Todo ello ha permitido conocer la percepción que tienen del SEO los profesionales de 
RLCS, Revista Latina de Comunicación Social, 79, 27-45 [Investigación] https://www.doi.org/10.4185/RLCS-2020-1487| ISSN 1138-5820 |Año 2021

este sector, identificar las posibilidades de su aplicación en la producción de noticia y proponer un marco para la ubicación del SEO en un diario digital.

PALABRAS CLAVE: Cibermedios; SEO; posicionamiento en buscadores; periodismo online; visibilidad web.

\begin{abstract}
Introduction. The importance that media professionals give to search engine positioning in journalistic writing and its different dimensions is analyzed. Specifically, the perception of journalism professionals is studied after the recent application of Search Engine Optimization (SEO) strategies in the Diario de Sevilla, a Joly Group cybermedia, with a tradition on paper and that, as of May 2018, created a specific web positioning department that would be its pilot project for the gradual transformation of the rest of its headers. Methodology. For the development of this research, participant observation, semi-structured interviews, and frequency analysis of terms from the company's internal manual on search engine positioning strategies have been applied. Conclusions. All this has made it possible to know the perception that professionals in this sector have of SEO, identify the possibilities of its application in news production, and propose a framework for the location of SEO in a digital newspaper.
\end{abstract}

KEYWORDS: Digital news media; SEO; Search Engine Optimization; Online journalism; website visibility.

\title{
CONTENIDO
}

1. Introducción. 2. Marco Teórico. 3. Metodología. 4. Resultados. 5. Discusión y conclusiones. 6. Referencias.

\section{Introducción}

La mayor parte de medios de comunicación cuentan con versiones digitales. Esto ha propiciado que los cibermedios se deban considerar sistemas con cualidades y necesidades específicas, tanto dentro del mundo del periodismo como dentro del ecosistema digital (Díaz-Noci y Salaverría, 2003, Rodríguez-Martínez et al, 2010).

A estas cualidades se unen las características intrínsecas de cada empresa periodística tanto por sus recursos técnicos y económicos (Iglesias-García, 2012) como por otras circunstancias, como la influencia de su edición impresa o la necesidad de una formación tecnológica adecuada de sus periodistas (Salaverría et al., 2018, Rodríguez-Martínez et al, 2010; Suárez-Sucre, 2017),

En este sentido, a despecho de las diferencias lógicas entre cada empresa, los redactores de los cibermedios necesitan añadir a su competencia una adecuada comprensión de las características del ecosistema digital, a fin de poder aplicarlas a la producción de la noticia (López-García et al., 2017; Muerza, 2017).

Uno de los condicionantes del medio digital es que cada vez es más importante el posicionamiento en buscadores o Search Engine Optimization (SEO) en la labor de las redacciones. La razón es que el SEO mejora la visibilidad de las noticias y por tanto aumenta las posibilidades de aparecer en los lugares preferentes de los resultados de búsqueda de Google y Bing, entre otros (Richmond, 2008; Dick, 2011). 
RLCS, Revista Latina de Comunicación Social, 79, 27-45 [Investigación] https://www.doi.org/10.4185/RLCS-2020-1487| ISSN 1138-5820|Año 2021

Esto se traduce en la obtención de mayor tráfico, lo que a su vez propicia la adquisición de nuevos lectores, objetivo que está en el núcleo de cualquier modelo de negocio de los medios de comunicación. Por tanto, el ecosistema digital ha implantado de forma decisiva la importancia económica y estratégica del SEO para la viabilidad de los cibermedios.

Por si fuera poco, además de motivos económicos, se puede argumentar que la unión del SEO con el buen periodismo ayuda a la existencia de ciudadanos activos e informados, lo que repercute en la salud democrática de una nación. El motivo de esta relación es que el SEO puede ayudar a posicionar información periodística de alta calidad y profesional y contribuir así a la libertad de prensa y a la democracia.

Es ante todas estas circunstancia que nace la motivación para este estudio centrado en el proceso de implementación relativamente reciente (mayo de 2018) del SEO en la redacción del Diario de Sevilla.

En concreto, para los autores, la oportunidad de estudiar este cibermedio viene justificada por tratarse de una cabecera de tradición impresa que, como se ha señalado, ha llevado a cabo la implantación reciente del posicionamiento en buscadores en su redacción. Además, esta experiencia inicial se ha llevado con el objetivo de su transferencia al resto de periódicos que integran el Grupo Joly. Se puede señalar, por tanto, que en la selección de este caso se han podido aplicar criterios de oportunidad y de máxima significación (Yin, 2014).

A partir de lo anterior, el enunciado del objetivo principal de este trabajo es el siguiente:

- Conocer la percepción de los profesionales del sector periodístico en relación con la implantación de una nueva estrategia de posicionamiento en buscadores para optimizar la visibilidad de sus noticias.

Adicionalmente a este objetivo, se planteó el siguiente objetivo derivado:

- Determinar las dimensiones de aplicación de estrategias SEO en la redacción periodística de un medio de comunicación diario de uno de los principales grupos de comunicación españoles.

Los apartados siguientes están organizados de este modo: en primer lugar, se presenta el marco teórico que se ha utilizado, después la metodología, seguida por los resultados para terminar con la discusión y las conclusiones.

\section{Marco teórico}

La presencia de la prensa en el ecosistema digital está en creciente aumento, hasta el punto en el que, en la actualidad, es muy difícil encontrar diarios en papel que no cuenten con versión online (Salaverría et al., 2018). Los actuales cibermedios, con ya dos décadas de historia tanto en el caso español (Salaverría et al., 2018) como a nivel internacional, han tenido que organizarse siguiendo una situación comunicativa diferente a las que afectaba en el pasado a los medios de comunicación tradicionales, principalmente debido a los procesos de interactividad (Cebrián-Herreros, 2009; Rodríguez-Martínez et al., 2012; Caminero y Sánchez-García, 2018) y a los cambios tecnológicos constantes que se producen en Internet.

Estos cambios están obligando a los cibermedios a reorientar sus contenidos y estrategias (Rodríguez-Martínez et al. 2010; Díaz-Noci, 2018; García-Avilés et al., 2018) con mucha rapidez (Suárez-Sucre, 2017). A su vez, esta celeridad está provocando cierta incertidumbre para los 
RLCS, Revista Latina de Comunicación Social, 79, 27-45 [Investigación] https://www.doi.org/10.4185/RLCS-2020-1487| ISSN 1138-5820|Año 2021

periodistas, que sienten que deben preservar los elementos y valores fundamentales del periodismo, al mismo tiempo que deben conocer adecuadamente las tecnologías que afectan a sus noticias en la web (López-García, 2017). Evidentemente, la integración de las redacciones al mundo online se ha convertido en una de las estrategias más importantes para los medios (Muerza, 2017), a esto hay que sumarle la importancia creciente de las redes sociales como un nuevo contexto para la mejora de la visibilidad web, la dinamización de las noticias (Trillo-Domínguez y Ollero, 2018).

En síntesis, la necesidad de la visibilidad web ha hecho que los medios de comunicación deban obtener un buen posicionamiento en buscadores (Carlson, 2007; Norris, 2007; Smyrnaios y Rebillard, 2009; Smyrnaios, 2015), ya que una parte del tráfico que reciben los cibermedios proviene de búsquedas de los usuarios en plataformas como Google (Norris, 2007; Machill et al., 2008; Giomelakis y Veglis, 2015).

Por este motivo, resulta esencial llevar a cabo estrategias SEO en las redacciones (Smyrnaios y Sire, 2014;), entendiéndose como SEO a las técnicas o procedimientos aplicados a sitios web que ayudan a incrementar las posibilidades de aparecer en los principales resultados de búsqueda (GonzaloPenela et al., 2015) y con ello tener mayor visibilidad y por tanto más lectores.

Como es lógico, en su detalle fino, las técnicas o estrategias SEO pueden diferir de un medio de comunicación a otro (Dick, 2011; Giomelakis y Veglis, 2015a; García-Carretero et al. 2016), principalmente porque cada medio cuenta con una serie de recursos de tiempo, financieros, técnicos y de apoyos gerenciales diferenciados (Dick, 2011). No obstante, no cabe duda y así lo expresan diversos estudios, de que la convergencia entre los principios periodísticos y los principios SEO han favorecido a las cabeceras (Asser, 2012; Lopezosa, Codina y Pérez-Montoro, 2019). Uno de los primeros casos de éxito que respaldan esta afirmación se encuentra en el portal web de $B B C$ News, que consiguió alcanzar las primeras posiciones en los resultados búsqueda con una estrategia SEO a partir de la segunda mitad de 2009. Esto provocó que, desde esa fecha hasta la primera mitad de 2011, las visitas a la sección noticias de BBC News aumentaran un 57\% y las visitas en conjunto de toda la web creciesen un 34\% (Asser, 2012; Newman y Levy, 2014).

Ante la confirmación del papel fundamental de la aplicación del SEO en los cibermedios como una parte del compromiso del medio con sus lectores (Richmond, 2008; Iglesias-García y Codina, 2016), se han asimilado algunas recomendaciones por parte de investigadores. A continuación, se destacan las principales estrategias SEO que los expertos en este campo consideran como adecuadas dentro de las redacciones de los cibermedios atendiendo al mayor consenso de técnicas identificadas de dichos autores y que se recogen en nuestra revisión bibliográfica.

- A nivel general se recomienda redactar noticias con la mayor calidad posible (Wordtracker, 2018), enriquecerlas con enlaces externos que apunten a fuentes de información de referencia (Graham Charlton, 2016), agruparlas temáticamente mediante taxonomías para que los buscadores las puedan identificar semántica con mayor precisión (Smyrnaios y Sire, 2014; Lopezosa, Codina y Pérez-Montoro, 2019) y dar acceso a los lectores para que puedan comentarlas y generar debate sobre ellas (Minos-Athanasios et al. 2019).

- Sobre el titular se aconseja aplicar el principio de la doble titulación. Se trata de una titulación pensada para el lector (titular periodístico) y otra para el buscador (titular SEO) (Asser, 2012). Adicionalmente, que al menos el titular SEO contenga palabras clave al principio del mismo (García-Orosa y López, 2014).

- En relación con el cuerpo de la noticia se recomienda que contenga al menos 300 palabras (Pérez-Montoro y Codina, 2017), contenido multimedia como fotografías y vídeos (Giomelakis y 
RLCS, Revista Latina de Comunicación Social, 79, 27-45 [Investigación] https://www.doi.org/10.4185/RLCS-2020-1487| ISSN 1138-5820 |Año 2021

Veglis, 2015; 2015 ) y un primer párrafo que incorpore palabras clave y sinónimos de las mismas (Codina et al. 2016).

- En cuanto a la optimización de la visibilidad de formatos en vídeo, se prioriza su SEO para el canal de YouTube del diario digital, atendiendo principalmente al uso estratégico de etiquetas y palabras clave dentro de los campos de descripción del vídeo (Lopezosa, Orduna-Malea, PérezMontoro, 2019).

- Asimismo, para poder comprobar la eficacia de la noticia publicada en cuanto a su visibilidad web se aconseja utilizar herramientas de auditoría SEO y aplicar medidas correctivas para mejorar su posicionamiento si fuera necesario

- Por último, se recomienda formar a los futuros periodistas en las diferentes facetas de SEO y analítica web para que de este modo tengan las herramientas necesarias para afrontar la lucha por las audiencias (Lopezosa et al. 2020).

Como se ha podido constatar, existen diversas recomendaciones más o menos establecidas sobre posibles técnicas SEO en cibermedios. Sin embargo, no se ha trabajado hasta la fecha la importancia que le dan los periodistas al posicionamiento en buscadores tras la reciente aplicación de estrategias de SEO y atendiendo a los posibles problemas que pueden surgir ya sea por el desconocimiento por parte de los periodistas sobre el posicionamiento en buscadores, o las propias rutinas periodísticas de un cibermedio de tradición en papel, etc.

Además, ninguno de estos estudios mencionados incluye la triangulación de técnicas que incluyen, al mismo tiempo la observación participante, entrevistas a profesionales implicados y el análisis cualitativo del manual SEO de una redacción periodística como parte de su metodología. Bajo esta importante premisa, se ha considerado la enorme conveniencia de poder realizar un estudio sobre SEO aplicado a Diario de Sevilla, que recientemente inició un proceso de total implantación de estrategias SEO en su redacción.

\section{Metodología}

Para llevar a cabo este estudio se ha utilizado, en primer lugar, el estudio de caso (Yin, 2014), concretamente del equipo de redacción del Diario de Sevilla. Para una adecuada triangulación, se ha llevado a cabo una observación participante, una batería de entrevistas semiestructuradas y un análisis cualitativo del contenido del manual interno sobre SEO del Grupo Joly, grupo mediático al que pertenece Diario de Sevilla.

Sobre el caso de estudio, se ha de señalar que el Grupo Joly cuenta con nueve periódicos: Diario de Cádiz, Diario de Jerez, Europa Sur, Diario de Sevilla, El Día de Córdoba, Huelva Información, Granada Hoy, Málaga Hoy y Diario de Almería. Asimismo, en su conjunto cuenta con 500.000 lectores y una venta diaria de 100.000 ejemplares en promedio. Por su parte, Diario de Sevilla es la primera cabecera de este Grupo mediático con una media de 70.000 lectores diarios. Una vez presentado el caso, se detalla, en lo que sigue, el proceso de trabajo seguido.

En lo que sigue, se explica con mayor detalle el diseño de la observación participante, para la cual se ha seguido a Angrosino (2012) y a Pons y Monistrol (2017); la configuración de las entrevistas semiestructuradas y los pasos dados para el análisis de contenido del manual interno de SEO del Grupo Joly.

En concreto para presentar el esquema de la observación participante en la tabla 1 se ha seguido la propuesta Pons y Monistrol (2017). 
RLCS, Revista Latina de Comunicación Social, 79, 27-45 [Investigación] https://www.doi.org/10.4185/RLCS-2020-1487| ISSN 1138-5820 |Año 2021

Tabla 1. Elementos estratégicos para el desarrollo de la observación participante en Diario de Sevilla.

\begin{tabular}{|c|c|}
\hline \multicolumn{2}{|c|}{ Facetas de la observación participante en Diario de Sevilla } \\
\hline ¿Qué investigar? & $\begin{array}{l}\text { Objetivo: Cómo es el trabajo SEO de los periodistas dentro de } \\
\text { un cibermedio y su grado de conocimiento de las técnicas de } \\
\text { este. } \\
\text { Foco de observación: Triple. Trabajo del departamento SEO de } \\
\text { un cibermedio (1). Trabajo SEO de los periodistas de la edición } \\
\text { digital (2). Trabajo SEO de una sección impresa que se está } \\
\text { adaptando a la edición digital (3). } \\
\text { Escenario de la observación: la redacción de Diario de Sevilla. }\end{array}$ \\
\hline ¿Cómo observar? & $\begin{array}{l}\text { Se llevó a cabo una observación participante, es decir, el } \\
\text { observador se integró como parte de la realidad que pretende } \\
\text { observar, en este caso, la redacción de Diario de Sevilla. } \\
\text { Todos los participantes supieron que estaban siendo observados. } \\
\text { Los participantes poseían plena información sobre el objeto de } \\
\text { observación. }\end{array}$ \\
\hline ¿Dónde observar? & $\begin{array}{l}\text { La redacción de la edición digital, la sección Actualidad } \\
\text { (sucesos) que trabajan tanto la versión digital como la edición } \\
\text { impresa del periódico y el departamento de SEO. }\end{array}$ \\
\hline ¿Qué describir? & $\begin{array}{l}\text { La observación se llevó a cabo con base en los objetivos del } \\
\text { estudio que se han indicado en la introducción. }\end{array}$ \\
\hline ¿Cuándo y cuánto observar? & $\begin{array}{l}\text { En septiembre de 2018. Durante una semana ocupando jornadas } \\
\text { laborales de } 9: 00 \text { a } 14.00 \text { y 16:00 a 18:00. }\end{array}$ \\
\hline ¿Cómo registrar? & $\begin{array}{l}\text { El registro se llevó a cabo en papel por parte de una persona. El } \\
\text { producto del análisis permitió visualizar los ámbitos y contextos } \\
\text { observados y su interpretación. }\end{array}$ \\
\hline
\end{tabular}

Fuente: Elaboración propia.

Además, para complementar el estudio de observación participante se llevaron a cabo 10 entrevistas semiestructuradas (Coller, 2000; Valles, 2002) a trabajadores de Diario de Sevilla.

A continuación, en la tabla 2 se muestran las preguntas de la entrevista y los objetivos específicos que se querían resolver con cada una de ellas.

Tabla 2. Construcción de las preguntas para las entrevistas semiestructuradas a trabajadores de Diario de Sevilla.

Tabla de metadatos de la entrevista

Objetivo

Pregunta 
RLCS, Revista Latina de Comunicación Social, 79, 27-45 [Investigación] https://www.doi.org/10.4185/RLCS-2020-1487| ISSN 1138-5820 | Año 2021

\begin{tabular}{|c|c|}
\hline $\begin{array}{l}\text { Conocer la percepción de los profesionales que } \\
\text { trabajan en medios de comunicación sobre qué } \\
\text { es el SEO. }\end{array}$ & ¿Cómo definiría el SEO? \\
\hline $\begin{array}{l}\text { Averiguar qué importancia otorgan los } \\
\text { profesionales del sector periodístico al SEO en } \\
\text { el seno de las redacciones. }\end{array}$ & $\begin{array}{l}\text { ¿Considera que es importante utilizar estrategias } \\
\text { SEO dentro de las redacciones de un periódico } \\
\text { digital? ¿Podría Justificar su respuesta? }\end{array}$ \\
\hline $\begin{array}{l}\text { Confirmar si los periodistas disponen de } \\
\text { formación sobre SEO y saben cómo aplicarlo a } \\
\text { las noticias. }\end{array}$ & $\begin{array}{l}\text { ¿Considera que los periodistas tienen información y } \\
\text { pautas suficientes para poder crear noticias } \\
\text { optimizadas para el SEO? }\end{array}$ \\
\hline $\begin{array}{l}\text { Concretar las técnicas SEO que conocen y } \\
\text { cuáles aplican cuando redactan sus noticias. }\end{array}$ & $\begin{array}{l}\text { ¿Qué estrategias SEO aplica cada día dentro de sus } \\
\text { rutinas productivas como periodista? }\end{array}$ \\
\hline $\begin{array}{l}\text { Saber si los profesionales de los medios de } \\
\text { comunicación consideran el SEO como parte } \\
\text { intrínseca de su trabajo o como una parte } \\
\text { marginal del mismo. }\end{array}$ & $\begin{array}{l}\text { ¿Cree que el periodista debería ser capaz de llevar a } \\
\text { cabo estrategias SEO o por el contrario estas } \\
\text { estrategias deberían depender del departamento de } \\
\text { posicionamiento web? ¿Podría justificar su } \\
\text { respuesta? }\end{array}$ \\
\hline $\begin{array}{l}\text { Conocer si los profesionales del sector } \\
\text { periodístico identifican el SEO como un } \\
\text { elemento de buena o mala calidad periodística. }\end{array}$ & $\begin{array}{l}\text { ¿Cree que afecta a la calidad de las noticias y al } \\
\text { servicio social de las mismas el tener que llevar a } \\
\text { cabo estrategias SEO? ¿Desea argumentar su } \\
\text { respuesta? }\end{array}$ \\
\hline $\begin{array}{l}\text { Desgranar la percepción de estos profesionales } \\
\text { sobre el futuro de los cibermedios en el contexto } \\
\text { de la visibilidad web. }\end{array}$ & $\begin{array}{l}\text { ¿Cómo ve el futuro del periodismo y su relación } \\
\text { con el SEO? }\end{array}$ \\
\hline
\end{tabular}

Fuente: Elaboración propia.

Por su parte, para seleccionar a los participantes de las entrevistas se tuvieron en cuenta las preguntas criterio (Valles, 2002) en donde se plantea quién tiene la información relevante, quién es más accesible social y físicamente, quién está dispuesto a informar y cooperar más y quién tiene una mayor fluidez comunicativa para informar con precisión. En este sentido, se muestra, en lo que sigue, los diferentes participantes de las entrevistas semiestructuradas y sus puestos dentro de Diario de Sevilla.

Tabla 3. Trabajadores de Diario de Sevilla entrevistados.

\begin{tabular}{|l|l|}
\hline \multicolumn{2}{|c|}{ TRABAJADORES DE DIARIO DE SEVILLA ENTREVISTADOS } \\
\hline \multicolumn{1}{|c|}{ Participante } & \multicolumn{1}{c|}{ Sección/departamento } \\
\hline Cristina Valdivieso & Periodista de la edición digital \\
\hline Manuel Rodríguez & Periodista de la edición digital \\
\hline Tamara Velázquez & Periodista de la edición digital \\
\hline Francisco Barquilla & Responsable del SEO del diario \\
\hline María Maldonado & Responsable de Marketing digital del diario \\
\hline Javier Salcedo & Jefe de producto \\
\hline
\end{tabular}


RLCS, Revista Latina de Comunicación Social, 79, 27-45 [Investigación] https://www.doi.org/10.4185/RLCS-2020-1487| ISSN 1138-5820 | Año 2021

\begin{tabular}{|l|l|}
\hline Fernando Pérez Ávila & Periodista sección actualidad (sucesos) \\
\hline Juan Manuel Marqués & Subdirector y responsable de la sección Política \\
\hline Alberto Grimaldi & Responsable y periodista de la sección Economía \\
\hline Rocío Mesa & Directora de Sistemas \\
\hline
\end{tabular}

Fuente: Elaboración propia.

Para analizar la información obtenida de las entrevistas, se utiliza la herramienta de análisis Nvivo. Se trata de un software que permite estructurar y categorizar ideas, analizar frecuencia de palabras de documentos, crear árboles de palabras, etc. En la actualidad, está considerada una de las herramientas más utilizadas para conocer y analizar correctamente el contenido de entrevistas y contenido en general.

A continuación, se describen los principales componentes y elementos de Nvivo y se concluye con su adaptación a las entrevistas realizadas.

Tabla 4. Tabla descriptiva de los principales datos que se pueden usar para el análisis cualitativo con Nvivo.

\begin{tabular}{|l|l|}
\hline \multicolumn{2}{|c|}{ Tipos de datos Nvivo } \\
\hline Tipo de dato & Descripción \\
\hline Recursos & Archivo editable de texto en otro formato. \\
\hline Memos & Anotaciones. \\
\hline Nodos o códigos & $\begin{array}{l}\text { Pueden ser temas, tópicos, conceptos, permite enlazar los materiales y poder } \\
\text { encontrarlos. Para codificar se cuenta con nodos libres (sin organización previa), } \\
\text { nodos de árboles (categorías o subcategorías) y nodos de caso (para diferenciar casos } \\
\text { y hacer preguntas). }\end{array}$ \\
\hline Atributos & $\begin{array}{l}\text { Son las propiedades que tienen los documentos y los nodos. Se puede utilizar Word, } \\
\text { pdf, hojas de cálculo, materiales de audio y video, páginas web y artículos en línea. }\end{array}$ \\
\hline
\end{tabular}

Fuente: Elaboración propia.

Para la interpretación de los datos de las entrevistas se generaron los siguientes nodos o códigos que se muestran a continuación:

- Calidad de noticias y SEO

- Definición SEO

- Formación SEO para periodistas

- Futuro del SEO en relación con el periodismo

- Importancia del SEO en la redacción periodística

Gracias a estos nodos se pudieron unificar las declaraciones de los diez entrevistados y dar respuestas solventes e integrales a las preguntas realizadas.

Una vez concluida nuestra observación participante y las entrevistas, pasamos a analizar el contenido del manual SEO que tienen todos los periodistas de este medio para aplicar a sus noticias. Para ello, realizamos un análisis de redes semánticas por repetición de términos, utilizando la herramienta NVivo. El manual analizado está creado por el SEO del grupo Joly, en él se explica qué es el SEO, su importancia para visibilidad de noticias, e incluye los principales elementos que un periodista debe optimizar y la manera de optimizar. Una vez incorporado este manual a NVivo, llevamos a 
RLCS, Revista Latina de Comunicación Social, 79, 27-45 [Investigación] https://www.doi.org/10.4185/RLCS-2020-1487| ISSN 1138-5820 |Año 2021

cabo una consulta de frecuencias de palabras. Esto nos muestra la frecuencia de uso de palabras dentro del documento interno SEO destacando las estrategias más importantes descritas en el mismo.

\section{Resultados}

A lo largo de esta investigación, se han abordado las principales dimensiones que intervienen en la implantación de un modelo de optimización de posicionamiento en buscadores en un cibermedio. Esto se ha llevado a cabo tanto desde el punto de vista del redactor/periodista como del experto en posicionamiento en buscadores.

Para ello, ha sido necesario investigar primero estos elementos por separado, para así entender cada una de las necesidades propias del SEO y del periodismo y después unirlas de forma armónica para que actúen de manera adecuada dentro de una empresa periodística.

De este modo, la observación participante permitió confirmar que, para implantar de manera integral el SEO de una redacción, es preciso separar claramente el trabajo del periodista (redactor) y el trabajo del experto en posicionamiento en buscadores (a cargo del Departamento SEO) puesto que sus funciones y aptitudes son muy diferentes.

Como resultado, se identificaron dos niveles de SEO muy significativos: (1) el nivel estratégico y dependiente del departamento de SEO que afecta de manera global al medio de comunicación online, y (2) el nivel táctico que recae directamente en los redactores y que afecta a las noticias y producción periodística del cibermedio.

En lo que sigue, se muestra de manera ampliada los dos niveles de SEO en forma de tabla de síntesis.

Tabla 5. Tabla con los niveles del SEO en cibermedios y sus funciones.

\begin{tabular}{|c|c|}
\hline & S DEL SEO EN CIBERMEDIOS Y SUS FUNCIONES \\
\hline $\mathrm{Niv}$ & Departamento SEO - Nivel estratégico - Conjunto del medio de comunicación \\
\hline ID & Funciones del departamento SEO \\
\hline 1 & $\begin{array}{l}\text { Implementar y seguir los esquemas de lenguajes de metadatos, como los de la norma } \\
\text { Schema.org promovida por los principales buscadores en el código fuente de la noticia, lo que } \\
\text { requiere configuraciones a nivel del CMS del medio. }\end{array}$ \\
\hline 2 & $\begin{array}{l}\text { Implementar y seguir la tecnología AMP y otras que puedan aparecer en el ámbito de la } \\
\text { publicación digital }\end{array}$ \\
\hline 3 & $\begin{array}{l}\text { Analizar y promover medidas para optimizar los resultados de búsqueda de las noticias } \\
\text { publicadas }\end{array}$ \\
\hline 4 & Mejorar la velocidad de carga de la web \\
\hline 5 & Optimizar la accesibilidad para todo tipo de lector \\
\hline 6 & $\begin{array}{l}\text { Optimizar el funcionamiento de los buscadores internos del cibermedio, así como la } \\
\text { arquitectura de la información del sitio web }\end{array}$ \\
\hline 7 & $\begin{array}{l}\text { Optimizar los vídeos subidos al canal YouTube (en el caso de que el cibermedio cuente con } \\
\text { uno) }\end{array}$ \\
\hline 8 & Realizar seguimientos de la competencia a nivel de palabras clave posicionadas \\
\hline 9 & Realizar seguimientos de la competencia a nivel de enlaces externos obtenidos \\
\hline 10 & $\begin{array}{l}\text { Realizar seguimiento de las publicaciones de la competencia para identificar noticias no } \\
\text { publicadas en su cibermedio y proponerlas a los periodistas para que creen nuevas noticias }\end{array}$ \\
\hline 11 & $\begin{array}{l}\text { Dar soporte y asesoramiento en la gestión de la venta de enlaces a terceros desde el punto de } \\
\text { vista de la coherencia y la transmisión de autoridad web (en caso de medios que tengan este } \\
\text { servicio implementado de forma transparente) }\end{array}$ \\
\hline 12 & $\begin{array}{l}\text { Analizar e identificar tendencias de búsqueda para ayudar a los redactores a encontrar temas } \\
\text { noticiosos }\end{array}$ \\
\hline 13 & Monitorizar redes sociales para identificar tendencias y temas para nuevas noticias (hashtags) \\
\hline
\end{tabular}


RLCS, Revista Latina de Comunicación Social, 79, 27-45

[Investigación] https://www.doi.org/10.4185/RLCS-2020-1487| ISSN 1138-5820 | Año 2021

\begin{tabular}{|c|c|}
\hline 14 & $\begin{array}{l}\text { Monitorizar y solucionar problemas técnicos de la web que afecten al SEO: errores de rastreo, } \\
\text { errores de carga, eliminar enlaces rotos, etc. }\end{array}$ \\
\hline 15 & $\begin{array}{l}\text { Desarrollar programas de formación interna sobre SEO a los periodistas de redacción (con } \\
\text { actualizaciones sobre los cambios en los motores de búsqueda) }\end{array}$ \\
\hline \multicolumn{2}{|r|}{ Nivel 2: Redactores - Nivel táctico - Para cada noticia o producción periodística } \\
\hline ID & Funciones del redactor a nivel de SEO de contenidos \\
\hline 1 & $\begin{array}{l}\text { Producir contenidos optimizados para la visibilidad web que mantengan un alto estándar de } \\
\text { calidad periodística }\end{array}$ \\
\hline 2 & $\begin{array}{l}\text { Redactar noticias que incorporen términos relacionados con la frase o palabra clave a } \\
\text { posicionar, siempre de un modo coherente con la buena escritura. }\end{array}$ \\
\hline 3 & Comprobar la presencia de la frase o palabra clave en el titular periodístico \\
\hline 4 & Comprobar la presencia de la frase o palabra clave en el titular SEO \\
\hline 5 & $\begin{array}{l}\text { Chequear y asegurar la densidad de la frase o palabra clave en el conjunto de la noticia, así } \\
\text { como su distribución en las secciones de la misma, como en los puntos } 1,2,3 \text { y } 4 \text {, siempre de } \\
\text { un modo coherente con la buena escritura periodística. }\end{array}$ \\
\hline 6 & $\begin{array}{l}\text { Asegurar el uso de una redacción rica en sinónimos y conceptos relacionados con la frase o } \\
\text { palabra clave }\end{array}$ \\
\hline 7 & $\begin{array}{l}\text { Incluir fuentes de información externas justificadamente relacionadas en el cuerpo de la } \\
\text { noticia (en su caso) }\end{array}$ \\
\hline 8 & $\begin{array}{l}\text { Incluir enlaces de noticias relacionados del mismo medio (en su caso) si la proximidad } \\
\text { temática de las noticias lo justifica. }\end{array}$ \\
\hline 9 & Incluir imágenes que acompañen a la noticia de modo justificado \\
\hline 10 & Incluir contenido audiovisual que ayude a entender mejor o que complete la noticia \\
\hline 11 & Utilizar adecuadamente enlaces internos y externos siempre que aporten valor al lector \\
\hline 12 & $\begin{array}{l}\text { Etiquetar temáticamente las noticias para que queden agrupadas utilizando una taxonomía de } \\
\text { modo coherente }\end{array}$ \\
\hline 13 & $\begin{array}{l}\text { Utilizar metadatos específicos (título y descripción) para las principales redes sociales, al } \\
\text { menos para Facebook y Twitter sin perjuicio de otras según el perfil del medio o de la noticia }\end{array}$ \\
\hline 14 & Difundir las noticias a través de las redes sociales del medio. Con el uso adecuado de hashtags \\
\hline 15 & $\begin{array}{l}\text { Crear piezas audiovisuales noticiosas y subirlas al canal de YouTube (en caso de que cuenten } \\
\text { con uno) }\end{array}$ \\
\hline 16 & $\begin{array}{l}\text { Publicar contenidos recomendados por } \\
\text { tendencias de búsqueda) }\end{array}$ \\
\hline
\end{tabular}

Fuente: Elaboración propia.

Respecto a la lista de los 16 puntos de chequeo del SEO de contenidos a cargo de los redactores cabe señalar dos cosas. Primero, es evidente que la lista podría ser de 14 o de 20 puntos, según la forma de presentar los mismos componentes. Segundo, por tanto, no se trata de que sean 16 o que sean 20, sino que estén bien definidos y apoyados en la máxima evidencia disponible, como se ha procurado hacer en este trabajo.

Una vez obtenidos los resultados de la observación participante, a continuación, se describen las principales aportaciones obtenidas de las entrevistas semiestructuradas, todas ellas centradas en conocer la asimilación estratégica tanto del departamento SEO como de la aplicación estratégica de técnicas de posicionamiento desde los diferentes departamentos de Diario de Sevilla, y la opinión de sus propios implicados.

Tabla 6. Síntesis de resultados de las 10 entrevistas semiestructuradas llevadas a cabo.

\begin{tabular}{|l|l|}
\hline Temas & Resultados \\
\hline Calidad de noticias y SEO & $\begin{array}{l}\text { En general, perciben el SEO como muy } \\
\text { importante en las redacciones ya que ayudan a }\end{array}$ \\
captar más lectores. Los entrevistados consideran \\
que el SEO no afecta a la calidad de las noticias. \\
Un porcentaje bajo considera que el SEO da un
\end{tabular}


RLCS, Revista Latina de Comunicación Social, 79, 27-45 [Investigación] https://www.doi.org/10.4185/RLCS-2020-1487| ISSN 1138-5820 |Año 2021

\begin{tabular}{|c|c|}
\hline & $\begin{array}{l}\text { valor añadido al contenido. Por último opinan que } \\
\text { aplicar estrategias sobre optimizadas de SEO a las } \\
\text { noticias (cosa que no ocurre en Diario de Sevilla) } \\
\text { haría que su calidad se vería mermada. }\end{array}$ \\
\hline Definición SEO & $\begin{array}{l}\text { Tienen un buen conocimiento sobre qué es el } \\
\text { SEO. Las declaraciones muestran dos esferas } \\
\text { sobre la definición del SEO. La primera hace } \\
\text { referencia al SEO como técnicas o prácticas para } \\
\text { que las noticias aparezcan en los primeros puestos } \\
\text { de Google. La segunda acepción sobre el SEO, } \\
\text { tiene que ver con el profesional que se encarga de } \\
\text { que las noticias se posicionen en Google. }\end{array}$ \\
\hline Formación SEO para periodistas & $\begin{array}{l}\text { Tienen formación suficiente sobre SEO, sin } \\
\text { embargo, al tratarse de una redacción con } \\
\text { tradición en papel, a veces cuesta entender el } \\
\text { cambio hacia el soporte digital. Los entrevistados } \\
\text { consideran que se está haciendo un gran esfuerzo } \\
\text { para formar a los periodistas en SEO. Se trata de } \\
\text { una formación SEO primaria basada } \\
\text { principalmente en la optimización de noticias y no } \\
\text { a estrategias técnicas complejas. Consideran que a } \\
\text { mayor formación sobre SEO mejor le irá a la } \\
\text { redacción. Por último, abogman por una } \\
\text { formación continua. }\end{array}$ \\
\hline Futuro del SEO en relación con el periodismo & $\begin{array}{l}\text { Consideran que el SEO bien aplicado no afecta a } \\
\text { la calidad de las noticias. De hecho, tienen la } \\
\text { creencia de que el futuro del periodismo pasa por } \\
\text { las plataformas digitales y en concreto por } \\
\text { Google, por lo tanto, las redacciones periodísticas } \\
\text { tenderán a buscar cada vez más una convergencia } \\
\text { entre periodismo y la visibilidad a través de } \\
\text { buscadores. }\end{array}$ \\
\hline Importancia del SEO en la redacción periodística & $\begin{array}{l}\text { Los entrevistados reconocen que el departamento } \\
\text { SEO en una redacción periodística es esencial y } \\
\text { que debe crearse una simbiosis entre la redacción } \\
\text { y este departamento. Por lo tanto deben ir unidos. } \\
\text { Los profesionales entrevistados entienden que } \\
\text { debe existir una visión externa, liderada por el } \\
\text { departamento SEO en la redacción. Este } \\
\text { departamento se encargaría de las estrategias y, en } \\
\text { cambio, los periodistas de redactar las noticias. El } \\
\text { responsable SEO daría las pautas básicas sobre } \\
\text { visibilidad web a los periodistas y también se } \\
\text { encargaría de revisar posibles errores de SEO. }\end{array}$ \\
\hline
\end{tabular}

Fuente: Elaboración propia.

Por último, se confirma que el manual interno de SEO para los trabajadores de Grupo Joly es una documentación que aborda las estrategias básicas que deben cumplir todas las noticias que se publiquen en la edición digital. Este manual se centra en el SEO de contenidos, es decir, en estrategias que hacen referencia al texto de la noticia, con especial énfasis en el uso de palabras clave (aquellas por las que el cibermedio aplica para que los lectores le encuentren en Google) en el titular, el cuerpo de la noticia, el cintillo y en la descripción de las imágenes de la noticia. También se recomienda aplicar la negrita y cursiva en estas palabras clave. Asimismo, se recomienda el uso de etiquetas/tags y enlaces en el cuerpo de la noticia que apunten a otras informaciones relacionadas. Adicionalmente, se confirma que el manual SEO debe estar vivo, porque las normas y características del posicionamiento en buscadores cambian muy a menudo y lo que hoy premia Google quizás no lo 
RLCS, Revista Latina de Comunicación Social, 79, 27-45 [Investigación] https://www.doi.org/10.4185/RLCS-2020-1487| ISSN 1138-5820 | Año 2021

haga en breve, sin embargo, al tratarse del primer manual de la reciente implementación del departamento SEO, todavía se desconoce cada cuánto lo revisarán y actualizarán.

\section{Discusión y conclusiones}

Como demuestra el estudio desarrollado en la redacción del Diario de Sevilla, apoyado en la triangulación metodológica que incluye observación participante, entrevistas semiestructuradas, así como el análisis de contenido del manual interno SEO de Grupo Joly, existen óptimas posibilidades de integrar de manera eficaz el posicionamiento en buscadores en una redacción periodística con amplía tradición en papel. A continuación, se muestra la discusión y conclusiones y para ello se revisan el objetivo principal y el secundario.

En primer lugar los resultados confirman que para implantar de manera integral el SEO de una redacción es preciso separar claramente el trabajo de los periodistas/redactores y el de los expertos o responsables SEO a nivel de todo el medio. Esta división se ha materializado en el Departamento de SEO, la aplicación del SEO por parte de los redactores del medio.

Al Departamento de SEO le corresponde una función estratégica de análisis y de generación de orientaciones para los redactores. Mientras que a la redacción le corresponde aplicar las recomendaciones de optimización SEO sin que las mismas afecten a la calidad periodística de las noticias.

Otra forma de entender esta distribución es la siguiente:

El departamento SEO: se encarga de las estrategias generales de SEO del medio, analiza la repercusión en los buscadores tras aplicar dichas estrategias, forma a los redactores, les da apoyo y se encarga de aplicar el SEO técnico que necesita el mantenimiento del portal.

Los redactores: deben conocer cómo afecta los resultados de búsqueda a sus noticias y a la obtención de más usuarios, entender el funcionamiento básico de los buscadores y aplicar las recomendaciones de visibilidad preparadas por el Departamento de SEO a sus noticias.

Este estudio confirma un claro interés por la optimización de las noticias en los buscadores por parte de los periodistas y un claro ejercicio práctico del SEO aplicado a las noticias, en cuanto a planificación y rutinas productivas. Asimismo, esta investigación obtiene resultados capaces de identificar las estrategias SEO más habituales que se pueden distribuir según sean para redactores o para el departamento de SEO. De hecho, el funcionamiento de la implementación de las estrategias SEO destaca en dos ámbitos, en la redacción de noticias por parte de los periodistas y la optimización estratégica global por parte del experto SEO. De hecho, desde la redacción y optimización de noticias se lleva a cabo el uso de palabras clave en el titular, la entradilla, la caja de texto, en las negritas, cintillo y en los pies de foto, etc., mientras que desde el departamento SEO se tiene un papel más estratégico centrado en el asesoramiento en posicionamiento en buscadores a todos los periodistas del diario e identificación de la búsqueda de tendencias de noticias para obtener más lectores.

Esta investigación corrobora que, en lo que respecta a implementación SEO de un diario con gran tradición en la edición impresa, existen posibilidades de poder llevarla a cabo de manera eficaz. Prueba de ello es la coherencia en las rutinas productivas de los principales actores que intervienen 
RLCS, Revista Latina de Comunicación Social, 79, 27-45 [Investigación] https://www.doi.org/10.4185/RLCS-2020-1487| ISSN 1138-5820 |Año 2021

en este proceso de implementación en este estudio de caso: departamento SEO, edición digital de Diario de Sevilla y secciones de papel/digital.

Si bien es cierto que el departamento SEO y el equipo de la edición digital (periodistas nativos digitales) tienen un mayor conocimiento sobre Internet y sobre el posicionamiento en buscadores, las secciones más tradicionales, aun con una mirada más condicionada por el formato papel, están siendo capaces de adaptarse de manera adecuada a la optimización de las noticias para aparecer en los buscadores.

Además, de los datos recogidos que se desprenden, la observación participante, las entrevistas y el análisis del manual SEO de Grupo Joly, se puede conocer perfectamente el funcionamiento de la redacción del cibermedio en el contexto de la visibilidad web.

Este funcionamiento se articula entre el departamento SEO como cabeza de las estrategias en posicionamiento en buscadores, los profesionales de la edición digital como editores de noticias y principales soportes para los periodistas de las demás secciones y los periodistas de las diferentes secciones que trabajan versión digital/versión impresa como los principales creadores de noticias de este medio de comunicación.

Por otra parte, en ocasiones se ha señalado que, aunque admiten formas de conciliación y, de hecho, así sucede de forma sistemática, los principios que rigen el buen SEO no siempre se apartan o no siempre coinciden con los de la redacción periodística. Esto es algo que, de forma prematura en la $B B C$ (Asser, 2012) se detectó y quedó más o menos bien solucionado con el principio de la doble titulación: la periodística, al cargo de la etiqueta h1 (en el código HTML) y la SEO, al cargo del metadato title (también en el código HTML).

La experiencia de esta investigación confirma que, efectivamente, se pueden conciliar, con ventaja mutua, los principios de calidad del mejor periodismo, con la optimización de la producción periodística para mejorar su visibilidad en el ecosistema digital.

Asimismo, se constata que los periodistas consideran que las estrategias SEO son esenciales en la redacción de un cibermedio como Diario de Sevilla, principalmente porque muchos lectores buscan la información a través de Google y el aparecer en los primeros resultados en los buscadores permite, no solo, competir por las mejores posiciones en los resultados de búsqueda, sino obtener mayor tráfico web en forma de nuevos usuarios.

De acuerdo con las anteriores aportaciones, se confirma el buen conocimiento de la misión del SEO en una redacción y su relación con la función de visibilidad de la producción periodística. Pese a lo que los diversos autores señalan (Suárez-Sucre, 2017; López-García, 2017; Muerza-Ferrer, 2017; Trillo-Domínguez y Ollero, 2018), se da el caso de redacciones donde se pueden unificar los intereses periodísticos como tales con los objetivos del SEO, al menos desde el importante punto de la percepción de los profesionales del sector periodístico.

Por último, se confirma que es factible generar una serie de recomendaciones SEO con alta transferibilidad a otros casos. En concreto, el caso revela que algunas de las mejores estrategias SEO se enmarcan en las siguientes dimensiones:

- Formación continua para los periodistas sobre SEO ya que esto les ayudará a comprender e interiorizar las nuevas rutinas productivas a las que se debe enfrentar un medio con gran tradición en la versión impresa. Se pone el énfasis en el aspecto de formación continuada porque las estrategias SEO sufren cambios y modificaciones frecuentes. 
RLCS, Revista Latina de Comunicación Social, 79, 27-45 [Investigación] https://www.doi.org/10.4185/RLCS-2020-1487| ISSN 1138-5820 |Año 2021

- Importancia del enlazado interno para reforzar la visibilidad y la autoridad de las noticias entre ellas.

- Uso de palabras clave y de etiquetas en secciones bien determinadas de las noticias: titular, cintillo, cuerpo y metadatos de las imágenes (en su caso), entre otros.

- Uso estratégico de YouTube (considerado el segundo buscador más importante después de Google) no solo como una herramienta para publicar vídeos y después añadirlos a las noticias, sino también llevar a cabo acciones de visibilidad web adicionales, ya que, al ser el segundo buscador con mayor tráfico web, permite aumentar las audiencias.

Se querría concluir esta investigación recordando que cada año aparecen nuevas acciones de emprendimiento en el mundo de los cibermedios. En general, se trata de empresas pequeñas que necesitan orientación sobre cómo conseguir el mejor encaje de su nuevo medio en el ecosistema digital.

Tanto los profesionales de los cibermedios como los estudiosos y académicos del periodismo necesitan aportaciones basadas en el estudio de casos reales. Cuando se analiza un caso concreto como este, no se puede pretender que su generalización esté garantizada, pero al presentarlo con el nivel de detalle que se ha procurado aportar, se asegura su plena transferibilidad, esto es, tanto otros profesionales como otros investigadores disponen ahora de elementos para decidir sobre su aplicación a casos similares, con aportaciones específicas que nunca antes se habían dado en la bibliografía especializada.

\section{Referencias}

Angrosino, M. (2012). Etnografía y observación participante en Investigación Cualitativa. Colección Investigación Cualitativa. Madrid, España: Editorial Morata

Asser, M. (2012). Search Engine Optimisation in BBC News. BBC. Consultado el 18 de enero de 2020. http://www.bbc.co.uk/blogs/internet/posts/search_engine_optimisation_in

Caminero, L. y Sánchez-García, P. (2018). El perfil y formación del ciberperiodista en redacciones nativas digitales, Hipertext.net, (16), 4-15. https://doi.org/10.31009/hipertext.net.2018.i16.04

Carlson, M. (2007). Order versus access: news search engines and the challenge to traditional journalistic roles, Media, Culture \& Society, 29 (6), 1014-1030. https://doi.org/10.1177/0163443707084346

Cebrián-Herreros, M. (2009). Nuevas formas de comunicación: cibermedios y medios móviles", Comunicar, 17(33). https://doi.org/10.3916/c33-2009-01-001

Charlton, G. (2016). How much do journalists and editors need to know about SEO? Search Engine Watch. Consultado el 20 de enero de 2020. https://searchenginewatch.com/2016/03/15/howmuch-do-journalists-and-editors-need-to-know-about-seo/

Codina, L., Iglesias-García, M., Pedraza, R., García-Carretero, L. (2016). Visibilidad y posicionamiento web de informaciones periodísticas: el framework SEO-RCP. Barcelona, España: Serie editorial DigiDoc-UPF. 
RLCS, Revista Latina de Comunicación Social, 79, 27-45 [Investigación] https://www.doi.org/10.4185/RLCS-2020-1487| ISSN 1138-5820 |Año 2021

Coller, X. (2000). Estudio de caso. Colección Cuadernos Metodológicos. Madrid: Centro de Investigaciones Sociológicas. https://bit.ly/2NjqLPZ

Díaz-Noci, J. (2018). Hipertexto en periodismo: realidad e investigaciones de futuro, Anuario ThinkEPI. 12, 230-235. https://doi.org/10.3145/thinkepi.2018.34

Díaz-Noci, J. y Salaverría, R. (2003). Manual de redacción ciberperiodística. Barcelona. Ariel. ISBN: 9788434412972

Dick, M. (2011). Search Engine Optimization in UK News Production, Journalism Practice, 5 (4), 462-77. https://doi.org/10.1080/17512786.2010.551020

García-Avilés, J., Carvajal, M., Arias, F. (2018). Implantación de la innovación en los cibermedios españoles: análisis de las percepciones de los periodistas, Revista Latina de Comunicación Social, 73, 369 - 384. https://doi.org/10.4185/RLCS-2018-1260

García-Carretero, L., Codina, L., Díaz-Noci, J., Iglesias-García, M. (2016). Herramientas e indicadores SEO: características y aplicación para análisis de cibermedios, El profesional de la información, 25 (3), 497-504. http://dx.doi.org/10.3145/epi.2016.may.19

García-Orosa, B. y López, X. (2014). Headlines in major European digital media in Europe: More functional than conceptual, Estudios sobre el Mensaje Periodístico, 21 (2), 833-847. http://dx.doi.org/10.5209/rev-ESMP.2015.v21.n2.50887

Giomelakis, D. y Veglis, A. (2015). Employing Search Engine Optimization techniques in Online News, Studies in Media and Communication, 3 (1), 22-33 http://dx.doi.org/10.11114/smc.v3i1.683

Giomelakis, D. y Veglis, A. (215a). Investigating Search Engine Optimization Factors in Media Websites, The case of Greece, Digital Journalism, 4 (3), 379-400 https://doi.org/10.1080/21670811.2015.1046992

Gonzalo-Penela, C., Codina, L., Rovira, C. (2015). Recuperación de información centrada en el usuario y SEO: categorización y determinación de las intenciones de búsqueda en la Web", Index comunicación, 5 (3), 19-27.

Iglesias-García, M. (2012). Rutinas productivas de un cibermedio nativo digital, Cuadernos de Información, 30, 9-20. https://doi.org/10.7764/cdi.30.424

Iglesias-García, M. y Codina, L. (2016). Los cibermedios y la importancia estratégica del posicionamiento en buscadores (SEO)”, Opción, 32 (9) 2016, 929-944

López-García, X., Rodríguez Vázquez, A., Pereira-Fariña, X. (2017). Technological Skills and New Professional Profiles: Present Challenges for Journalism, Comunicar, 25 (53), 81-90. https://doi.org/10.3916/C53-2017-08

Lopezosa, C., Orduna-Malea, E., Pérez-Montoro, M. (2019). "Making Video News Visible: Identifying the Optimization Strategies of the Cybermedia on YouTube Using Web Metrics", en Journalism Practice, 14:4, 465-482. https://doi.org/10.1080/17512786.2019.1628657 
RLCS, Revista Latina de Comunicación Social, 79, 27-45 [Investigación] https://www.doi.org/10.4185/RLCS-2020-1487| ISSN 1138-5820 | Año 2021

Lopezosa, Carlos., Codina, Lluís., Pérez-Montoro, Mario. (2019). "SEO and Digital News Media: Visibility of Cultural Information in Spain's Leading Newspapers", en Trípodos, n.44, pp.41-61.

Lopezosa, C., Codina, L., Díaz-Noci, J. y Ontalba, J. (2020). SEO and the digital news media: From the workplace to the classroom", Comunicar, 63, 65-75. https://doi.org/10.3916/C63-2020-06

Machill, M., Beiler, M., Zenke, M. (2008). Search-engine research: a European-American overview and systematization of an interdisciplinary and international research field, Sage Journals, 30(5), 591-608 https://doi.org/10.1177/0163443708094010

Minos-Athanasios, K., Evangelos, L., Matina, K., Nikos, A. (2019). SEO Practices: A Study about the Way News Websites Allow the Users to Comment on Their News Articles, Future Internet, 11, 188; https://doi.org/10.3390/fi11090188

Muerza Ferrer, A. (2017). Producción de noticias radiofónicas en el contexto de la convergencia periodística: análisis de Radio Marca y sus sinergias con Marca y marca.com, Estudios sobre el Mensaje Periodístico 24(1), 758-795. https://doi.org/10.5209/ESMP.59979

Newman, N., David-Levy, A. L. (2014). Reuters Institute Digital News Report 2014. Tracking the Future of News, Reuters Institute for the Study of Journalism, University of Oxford.

Norris, C. (2007). SEO For Journalists: Introduction \& Keywords. Moz. Consultado el 18 de enero de 2020. https://moz.com/ugc/seo-for-journalists-introduction-keywords-part-1-of-5

Pérez-Montoro, M. y Codina, L. (2017). Navigation Design and SEO for Content-Intensive Websites. A Guide for an Efficient Digital Communication. Oxford, Reino Unido: Chandos Publishing

Pons, M., Monistrol, O. (2017). Técnicas de generación de información en investigación cualitativa II. En: Calderón C, Conde F, Fernández de Sanmamed MJ, Monistrol O, Pons M, Pujol E, Sáenz de Ormijana A. Curso de Introducción a la Investigación Cualitativa. Máster de Investigación en Atención Primaria. Barcelona: semFYC. Universitat Autònoma de Barcelona. Fundació Doctor Robert.

Richmond, S. (2008). How SEO is changing journalism, British Journalism Review, 19. pp 5. https://doi.org/10.1177/0956474808100865

Rodríguez-Martínez, R., Codina, L., Pedraza-Jiménez, R. (2010). Cibermedios y web 2.0: modelo de análisis y resultados de aplicación, en El profesional de la información, 19(1), 35-44. https://doi.org/10.3145/epi.2010.ene.05

Rodríguez-Martínez, R., Codina, L., Pedraza-Jiménez, R. (2012). Indicadores para la evaluación de la calidad en cibermedios: análisis de la interacción y de la adopción de la Web 2.0, Revista española de documentación científica, 35(1), 61-93. https://doi.org/10.3989/redc.2012.1.858

Salaverría, R., Martínez-Costa, MP. y Breiner, J. (2018). Mapa de los cibermedios de España en 2018: análisis cuantitativo, Revista Latina de Comunicación Social, 73, pp. 1034 a 1053. https://doi.org/10.4185/RLCS-2018-1295 
RLCS, Revista Latina de Comunicación Social, 79, 27-45 [Investigación] https://www.doi.org/10.4185/RLCS-2020-1487| ISSN 1138-5820 |Año 2021

Smyrnaios, N. y Rebillard, F. (2009). L'actualité selon Google. L'emprise du principal moteur de recherche sur l'information en ligne, Communication et Langages, 160, 95-109. https://doi.org/10.4074/S0336150009002087

Smyrnaios, N. y Sire, G. (2014). The News according to Google How Does Algorithmic Infomediation Frame the Work of French Journalists?. JSS-ECREA 2014 Conference, Thessaloniki.

Smyrnaios, N. (2015). Google and the Algorithmic Infomediation of News, Media Fields Journal, 10, 1-10. http://mediafieldsjournal.squarespace.com/google-algorithmic-infomedia/

Suárez-Sucre, E. (2017). Comprendiendo a los cibermedios. Una propuesta estructural para el análisis de los medios de comunicación en la red, Estudios sobre el Mensaje Periodístico, 23(1), 273-288. http://dx.doi.org/10.5209/ESMP.55596

Trillo-Domínguez, M. y Ollero, J. (2018). Estrategias de comunicación periodística para publicar en Redes Sociales desde la prensa local: Plan de Acción de Grupo Joly, Hipertext.net, (16), 68-77. https://doi.org/10.31009/hipertext.net.2018.i16.11

Valles, M. (2002). Entrevistas cualitativas. Colección Cuadernos Metodológicos. Madrid, España: Editorial Centro de Investigaciones Sociológicas.

Wordtracker (2018). A guide to SEO for editorial teams and journalists". Wordtracker. https://www.wordtracker.com/academy/seo/getting-started/seo-for-editorial-teams-journalists

Yin Rober, K. (2014). Case study research. Design and methods. Canadá: SAGE. ISBN: 9781 452242569

\section{AUTORES:}

\section{Carlos Lopezosa}

Carlos Lopezosa es profesor asociado de la Universitat Pompeu Fabra de Barcelona. Imparte docencia en la Facultad de Comunicación, en el Grados de Periodismo y de Comunicación Audiovisual. Es coordinador y profesor del Máster Online en Buscadores y SEO/SEM de Barcelona School of Management (UPF). Es doctor en Ciencias de la Información y especialista en SEO y sistemas de monetización basados en estrategias de contenidos de calidad. Actualmente forma parte del Grupo de Investigación DigiDoc de la Universitat Pomepu Fabra.

carlos.lopezosa@upf.edu

Índice H: 6

Orcid ID: https://orcid.org/0000-0001-8619-2194

Google Scholar: https://scholar.google.es/citations?hl=es\&user=v8EFUW4AAAAJ

Research Gate: https://researchgate.net/profile/Carlos Lopezosa

Scopus ID: https://www.scopus.com/authid/detail.uri?authorId=57209369822

\section{Magdalena Trillo-Domínguez}

Magdalena Trillo-Domínguez es directora de Granada Hoy, Subdirectora de Grupo Joly y profesora asociada de Comunicación Periodística en el grado de Comunicación Audiovisual y en el Máster en Nuevos medios interactivos y periodismo multimedia, ambos de la UGR. Licenciada en periodismo por la Universidad de Málaga y doctora en comunicación por la Universidad de Granada, se ha 
RLCS, Revista Latina de Comunicación Social, 79, 27-45 [Investigación] https://www.doi.org/10.4185/RLCS-2020-1487| ISSN 1138-5820 | Año 2021

especializado en periodismo digital y gestión cultural por la UOC y forma parte del grupo de investigación Nar-Trans sobre narrativas y comunicación transmedia y colabora de forma habitual en los espacios de análisis y debate de actualidad de la RTVA.

mtrillo@ugr.es

Índice H: 4

Orcid ID: http://orcid.org/0000-0001-9505-1203

Google Scholar: https://scholar.google.es/citations?hl=es\&user=ToNk0nYAAAAJ

Research Gate: https://researchgate.net/profile/Magdalena-Trillo-Dominguez

Scopus ID: https://www.scopus.com/authid/detail.uri?authorId=24345339900

\section{Lluís Codina}

Lluís Codina es profesor de la Universitat Pompeu Fabra de Barcelona. Imparte docencia en la Facultad de Comunicación, en los Grados de Periodismo y de Comunicación Audiovisual. Es coordinador del Máster Universitario en Comunicación Social (MUCS) del Departamento de Comunicación. Forma parte del staff académico e imparte docencia también en los Másteres Universitarios Online en Documentación Digital y en Buscadores, del Instituto de Educación Continua. Es miembro promotor del Grupo de Investigación en Documentación Digital y Comunicación Interactiva (DigiDoc), y coordinador de su Seminario de Investigación.

lluis.codina@upf.edu

Índice H: 31

Orcid ID: https://orcid.org/0000-0001-7020-1631

Google Scholar: https://scholar.google.com/citations?user=89k7vMMAAAAJ\&hl=es

Research Gate: https://researchgate.net/profile/Lluis Codina2

Scopus ID: https://www.scopus.com/authid/detail.uri?authorId=23392068000

\section{Marga Cabrera Méndez}

Marga Cabrera es doctora en Comunicación Audiovisual por la Universitat Politècnica de València y profesora titular de asignaturas relacionadas con la comunicación digital desde 2003, tanto en grado como en postgrado. Es directora del máster en social media, master CALSI. Fundadora y codirectora del congreso Internacional Comunica2 sobre redes sociales. Coordinadora del libro de la Fundéu BBVA "Escribir para internet: guía para los nuevos medios y las redes sociales". Autora del libro de la UOC "Emprender en periodismo, herramientas para el nuevo profesional en red". Editora del libro "Digital Tools for Academic Branding and Self-Promotion". Actualmente se encuentra inmersa en su proyecto "Observatorio de nuevos medios en español: www.nuevosmedios.es" financiado por la Fundación BBVA, ahora becas Leonardo. Desde 2016, forma parte del Consejo Directivo de SembraMedia, organización sin ánimo de lucro con sede en Los Ángeles (EEUU) dedicada a promover el emprendimiento en medios nativos digitales de lengua hispana.

mcabrera@upv.es

Índice H: 8

Orcid ID: https://orcid.org/0000-0001-7164-1815

Google Scholar: https://scholar.google.es/citations?user=RK2LvbYAAAAJ\&hl=es

Research Gate: https://researchgate.net/profile/Marga_Cabrera

Scopus ID: $\underline{\text { https://www.scopus.com/authid/detail.uri?authorId=56111467200 }}$ 